\title{
A Study on the Middle-Aged Women's Perception on Facial Aging
}

\author{
Moon-sin Jung ${ }^{1}$ and Hye-jin Kwon ${ }^{2}$ \\ ${ }^{1}$ Dep.of Medical Beauty Idustry, Graduate School, Cha University, Gyeonggi-do, \\ Korea \\ ${ }^{2}$ Dep. of Chemitry Engineering, Soongsil University, Seoul, Korea \\ ${ }^{1}$ Moonsin@naver.com, ${ }^{2}$ Corresponding Author: kwonhj0070@ssu.ac.kr
}

\begin{abstract}
In this study, the survey was carried out targeting women residing in Seoul and Gyeonggido Area from August 1, 2015 to August 30, 2015 in order to examine the subjective perception and aging improvement desire of middle-aged women in 40s to 50s on facial skin aging. The survey result is as follows. First, the survey result showed that women whose average length of life became longer showed a very low subjective perception and a high degree of concern on their aging due to psychological anxiety caused by aging such as loss of femininity and youthfulness due to rapid physical changes including menopause. Second, most respondents showed a strong desire for improving their aging and they were willing to give up their wealth and length of life for delaying their aging. To sum it up, the improvement of facial skin aging gave a positive influence on middle-aged women who evaluated themselves negatively due to as decreased self-esteem, worthlessness and lack of confidence due to aging anxiety and the recovery of confidence through the appearance management also helped establishing the interpersonal relations and becoming an important means to recover from social pressures.
\end{abstract}

Keywords: Facial aging, Middle-aged women, Aging improvement, Beauty programs, Cosmetic products

\section{Introduction}

Middle-aged people today include active seniors originated from the phase 'Today's seniors are not yesterday's seniors' and active seniors refer to the middle class over the age of 50 who begin to spend on their health, beauty and self-development actively. The middle-aged people who intend to find their new identity as the prime time in their life are distinguished from the silver generation in the aspect that they are interested in their health and appearance, enjoy consumption and leisure life and actively participate in social activities and they are also active in planning and preparing their second life while making an investment in themselves which they were unable to do previously.

However, the middle-aged women who get out of rearing their child(ren) due to the growth and independence of their child(ren) experience self-esteem due to loss of rearing role and psychological anxiety accompanied by depression as well as anxiety for the retirement of their husband and old age, showing a difference in various complex psychological characteristics and they also start experiencing rapid physiological and outward aging so that a mid-life crisis to undergo pain with confusion in their identity and a feeling of helplessness to feel loss of goal in life may also begin. When the role for self-fulfillment is not granted

Article history:

Received (June 29, 2016), Review Result (August 01, 2016), Accepted (September 19, 2016) 
practically, they feel the loss of opportunity for a new life and face psychological distress so that they experience social and psychological crisis.

The middle-aged women who intend to conform their value and put meaning on the satisfaction of their desire for recognition and the establishment of social relations by participating social activities to overcome such psychological changes want an active method called social activities for the feeling of their own happiness that leads to the situation that they enjoy cultural life or hobbies. On one hand, the management of appearance for improving self respect and overcoming depression is necessary for middle-aged women and the maintenance of health and youth has become an important concern. They consider becoming beautiful as a social norm and pursue cultural stereotype on beauty endlessly so that the way of thinking that it is possible to promote the beauty of their appearance artificially has become more widespread. The appearance is one of very important elements for improve their body according to the agreed aesthetic sense under the culture of the period that they are living, and it also significantly affects the evaluation of their value. The middle aged-women who have a very strong desire and interest for managing their appearance under the domestic stability and good social position are becoming new major consumers in the beauty market and they also wish to develop healthy and beautiful appearance even using an artificial way to create favorable impression.

Although it is impossible to block skin aging due to physical aging, efforts for reducing skin aging and symptoms are being made continuously and the interest in skin aging continues to increase. Since the skin accounts for the largest area in terms of pursuing external health and beauty for maintaining the appearance beautifully, the middle-aged women give a very important meaning to the management of their appearance such as active responses to aging such as freckles, spots and wrinkles. They also focus on the fundamental improvement of skin while emphasizing naturalness rather than external beauty in terms of aesthetic aspect along with a new periodically topic of "Well-Being", so their professional skin management behaviors are expanded and their interest and consumption on skin care are also increasing. Also, since the number of people who wish to develop their appearance for their psychological satisfaction increases, more people visit cosmetic surgery and more people desires the natural improvement of their skin due to the spread of zero makeup trend, so the skin beauty industry is growing significantly while the segmentation and specialization are being emphasized in each field.

Therefore, the purpose of this study is to present aesthetic measures to allow middle-aged women who experience social sense of alienation and psychological anxiety due to their psychological, social and physiologic changes to recover their confidence and solve their anxiety for aging by investigating and analyzing the middle-aged women's subjective perception, satisfaction, skin care actions and improvement desires for facial skin aging.

\section{Theoretical background}

\subsection{Skin aging of middle-aged women}

Skin aging of middle-aged women indicates that their skin starts to lose vitality in the functional and aesthetic aspects. The causes for skin aging can be divided by the genetic factors and the external factors that are commonly and widely known. The first aging is aging due to the genetic program of individuals with advancing years, that is, intrinsic aging that occurs due to skin structure change and deteriorated physiologic function, and intrinsic skin aging is relatively minor and fine wrinkles, xeroderma, decreased elasticity and tension occur. 
The second aging is photoaging due to oxidant stress such as UV rays and external stimuli. Matrix metalloproteinases (MMPs), collagenase that increase due to repeated exposure to ultraviolet light, break down collagen on the skin and reactive oxygen species cause damage to DNA and the peroxidation of cell membrane lipid and destroy collagen and elastin, accelerating skin aging. When aging process continues, collagen and elastic fibers made up of fibrous tissues that play a role of support that holds the skin structure become hardened and insoluble and collagen protein molecules and elastic fibers that give elasticity to skin undergo crosslinking process. The substances filling up the spaces between cells, collagen and elastic fibers is called Groundsubstance which has excellent ability to hold moisture and such substances are reduced as aging process, creating wrinkling on the skin.

Especially, the middle-aged women experience rapid wrinkle increase due to the reduction of collagenesis due to estrogen deficiency after menostasia and middle-aged women's degree of risk for wrinkle occurrence is approximately 3.5 times higher than that of middle-aged men. Aged skin shows quantitative decease in keratinocyte lipid and the number of lipid double membranes between keratinocytes. Therefore, the cell division of the keratinocyte on the basal layer is deteriorated and the period required for turn-over becomes longer and a change in the lipid layer of skin barrier occurs accordingly and the role of barrier is carried out properly during normal state but the restoration ability is deteriorated after acute skin barrier damage, and melanic pigments are generated in the skin of middle-aged women exposed to sunlight for a long period of time due to various factors inside and outside the body, causing coloring [1][2][3][4][5][6][7][8].

\subsection{Middle-aged women's perception on aging}

The middle age period is the period to accept physical and psychological changes, look back upon the past, look far into the future in the middle of life and affirm important tasks to review one's life. Especially, middle-aged women whose length of life after their middle age became longer due to the average life extension face the turning point of their life where cultural pressures such as the culture that emphasizes youth, fast information circulation through Internet and increased leisure time, physical changes such as menostasia, various psychological and social changes such as growth and independence of their child(ren), retirement of spouse and change of their roles, loss of feminine attraction and youth due to rapid physiological changes such as hormone imbalance and experience the sense of alienation and depression due to loss of roles in the process to approach their old age.

\section{Method of study}

A preliminary survey was carried out targeting women in the age of 40 to 59 residing in Seoul and Gyeonggi-do from July 1, 2015 to July 30, 2015, and then the purpose of the survey was explained properly through direct interview and the self-administrated questionnaire was carried out in order to refer to various opinions, and the main survey was carried out from August 1, 2015 to August 30, 2015 after modifying and supplementing some questions as a result of the preliminary survey. The questionnaire consisted of general characteristics, subjective perception on their skin and desire for improving facial aging and the collected data was analyzed using the SPSS (Statistical Package for Social Science) v. 20.0 Statistical Package program through the data coding and data cleaning process for the statistics process. 


\section{Result and consideration}

In this study, it was intended to investigate the perception and improvement desire on facial skin aging targeting middle-aged women in Korea to see the effect of facial skin aging improvement on their anxiety for aging occurred due to the physical aging process.

The middle age and old age period of modern women became longer than in the past due to life extension and life-cycle change. Most middle-aged women begin to question who they are due to loss of rearing role when they reach 40s to 50s and they face serious anxiety state and psychological crisis for their confidence and self-efficacy caused by rapid physical aging. The middle-aged women who face anxiety state for aging that occurs in the aging process intend to pursue the restoration of confidence and happiness in their life by actively participating social activities and they also have a very strong desire and interest in managing their appearance and improving facial skin aging for beauty demanded by the society and self-satisfaction.

Therefore, as a result of examining the level of satisfaction and value that middle-aged women received through the improvement of facial skin aging and the influential level of such improvement, women showed a very strong desire for improvement and they answered "for self-satisfaction" mostly for the reason of their desire. Also, they were willing to give up their wealth and length of life to look younger than they were. This seems to be the expression of their desire to restore their youth lost due to physical aging such as menostasia and maintain the feminine attraction longer to live the rest of their life in healthy, beautiful and confident way.

Most of them desire to look 5 years younger than they are now but some respondents who have more concerns due to skin aging or think that aging is deteriorated due to stress desire to look 10 years younger, showing a relatively higher desire.

Also, the respondents were willing to give up 'less than 1 year' or 'less than 1-2 years' of their life if they could look 5 years younger than they were, and they were willing to give up a longer period as the desired life time was longer, the respondents who thought their skin was healthy showed their desire to look younger by giving up 'less than 1-2 years', and most respondents desired to look younger by giving up less than 3 million KRW out of their wealth and even some respondents showed their desire to live younger by giving up more than 10 million KRW if the desired life time was longer, and they expressed their desire for improving by giving up less than 3-5 million KRW if they were unsatisfied with their skin aging state. It is considered that their desire to be compensated for their physical loss and loss of confidence they experience while growing older due to the pressure created by the society where youth is emphasized and physical beauty of women is valued is expressed as the management of appearance.

Also, most respondents answered that the improvement of facial skin aging influenced the beginning of their 2nd life, restoration of their physical and mental confidence and change in the quality of their life, and according to the result of this study, the restoration of confidence obtained through the management of appearance also had a positive influence on the establishment of interpersonal relations with others, indicating that the improvement of facial skin aging affected the aesthetic aspect as well as social and psychological aspects.

\section{Conclusion}

This study aimed to present theoretical grounds required for the development of aging improvement program to solve physical, psychological and social anxiety of middle-aged 
women occurred in the aging process and helped the development of rational aesthetic program that allowed people to understand the purpose of aged skin improvement by middleaged women was their desire for pursuing beauty instinctively as well as an important means to overcome their anxiety for aging.

The meaningful results were drawn through this study. However, the following limitations are pointed out and the direction for follow-up studies in future is presented.

First, the target people and area for survey were limited to Seoul and Gyeonggi-do area in this study, so there is a limitation to expand and generalize the result of this study on a national scale and a careful consideration is required. It is necessary to carry out a study in future by expanding the target area and people.

Second, the middle-aged women's desire for improving their facial skin aging was investigated and examined in this study and it is necessary to carry out diverse studies regarding alternatives to realize their desires to draw extensive results and help the development of the relevant industry.

In summary, it would be possible to present preliminary data for alternatives to improve facial skin aging more clearly if a study is carried out in future by expanding the target area and people, considering various investigation methods and comparing between target people.

\section{References}

[1] Shaw RB Jr, Katzel EB, and Koltz PF, "Aging of the mandible and its aesthetic implications," The Journal of Plastic Reconstruction Surgery, International Information Institute, vol.12, no.1, pp.332-3422, (2010) DOI:10.1097/prs.0b013e3181c2a685

[2] Rexbye H, Petersen I, and Johansens M, "Influence of environmental factors on facial ageing," The Journal of Age Ageing, International Information Institute, vol.35, no.2, pp.110-115, (2006) DOI:10.1093/ageing/afj031

[3] Rinnerthaler M, Bischof J, and Streubel MK, "Oxidative stress in aging human skin," The Journal of Biomolecules, International Information Institute, vol.5, no.2, pp.549-589, (2015) DOI:10.3390/biom5020545

[4] Lamb and S., "Permanent personhood or meaningful decline Toward a critical anthropology of successful aging," The Journal of Aging Studies, International Information Institute, vol.29, pp.41-52, (2014) DOI:10.1016/j.jaging.2013.12.006

[5] Keane and W, "Freedom, reflexivity, and the sheer everydayness of ethics," Journal of Ethnographic Theory, International Information Institute, vol.4, no.1, pp.443-457, (2014)

[6] Kwack and JK, "Effects of dietary behavior on the skin aging and acne of middle-aged women," Konkuk University Press, pp.93-106, (2013)

[7] Kim and HJ, "The effects of self efficacy and social supports on adoption of anti-aging products - the mediating effect of aging anxiety," Sejong Press, pp.93-106, (2013)

[8] Cho and IY, "Research on sense of value and perception on successful aging of Korean middle aged people," University of Ewha Woman Press, pp.9-16, (2015) 


\section{Authors}

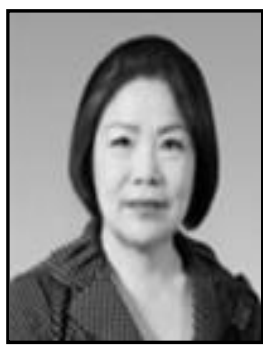

Moon-sin Jung

Adjunct Professor Dep. of Beauty Art, Seojeong Univ.

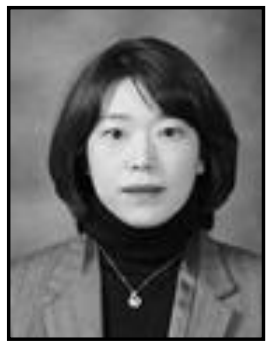

\section{Hye-jin Kwon}

Professor Dep. of Chemitry Engineering, Soongsil Univ. 